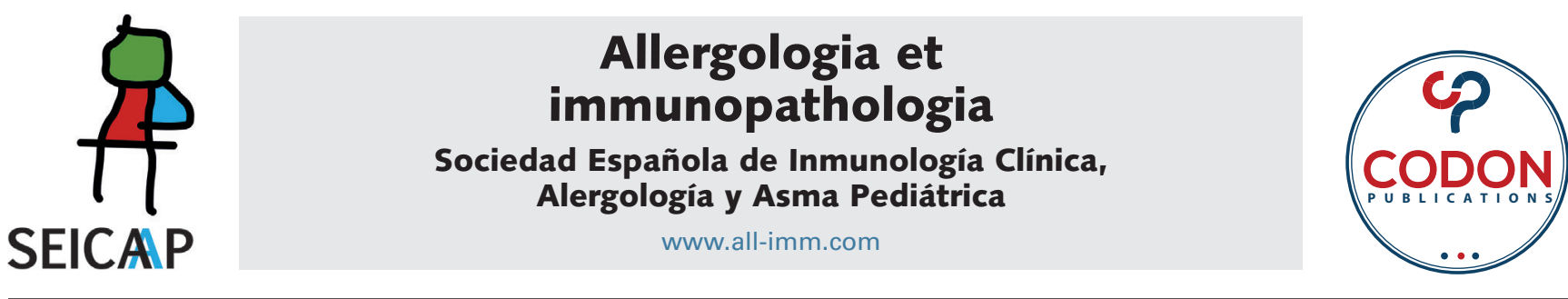

CASE SERIES

OPEN ACCESS (c) $\underset{\mathrm{BY}}{(\mathrm{NC} \text { () }}$

\title{
Allergic reactions to midazolam: A case series from an Italian allergy unit
}

\section{Eleonora Nucera ${ }^{\dagger}$, Giuseppe Parrinello $^{\dagger}$, Alessandro Buonomo, Arianna Aruanno*, Angela Rizzi}

\author{
Allergy Unit, Fondazione Policlinico Universitario A. Gemelli IRCCS, Rome, Italy \\ These authors contributed equally to this work.
}

Received 17 February 2021; Accepted 27 March 2021

Available online 1 July 2021

\section{KEYWORDS}

anaphylaxis; case

series; Kounis

syndrome; midazolam

hypersensitivity; skin tests

\begin{abstract}
Midazolam is a short-acting benzodiazepine with central nervous system depressing action, commonly used for conscious sedation for various procedures and for its pharmacologic properties.

In literature, severe adverse reactions to this drug are described, but only in few cases positive allergological tests were demonstrated.

The authors collected herein five clinical cases of different allergic reactions to midazolam demonstrated by positive skin tests.

The $1^{\circ}$ case is a suspected Kounis syndrome with cardiorespiratory arrest during an elective video laparoscopic cholecystectomy. The $2^{\circ}$ and $5^{\circ}$ cases are two systemic reactions with involvement of the skin and the gastrointestinal/respiratory system during elective surgeries in two patients with clinical history of atopia, while the $3^{\circ}$ and $4^{\circ}$ cases are local skin reactions in correspondence with the infusion site of midazolam during the execution of a colonoscopy.

All the patients performed a complete allergological evaluation for the reaction involved drugs. In all cases, only the intradermal test (IDT) with midazolam at $0.5 \mathrm{mg} / \mathrm{mL}$ was positive. Allergological tests performed in 10 healthy controls with negative results supported the diagnosis.

Therefore, midazolam is often considered a safe drug, because it does not have any active metabolites, in rare cases, it could cause different types of allergic adverse reactions: from severe anaphylaxis with cardiorespiratory arrest to simple local skin reactions.

Skin tests remain the first line in the diagnosis of an immediate-type hypersensitivity to midazolam; even if they could lose in sensitivity with increasing latency from the event. However the concentrations recommended by current guidelines of European Network for Drug Allergy (ENDA) and the European Academy of Allergy and Clinical Immunology (EAACl) drug allergy interest groups might not rule out some false-positive reactions due to an irritant effect that should be considered. In doubt cases, other allergological or laboratory tests (i.e., basophil
\end{abstract}

*Corresponding author: Arianna Aruanno, MD, PhD. Allergy Unit, Fondazione Policlinico Universitario A. Gemelli IRCCS, Largo F. Vito, 1 - 00168 Rome, Italy. Email address: arianna.aruanno@policlinicogemelli.it; aarianna@hotmail.it 
activation tests, serum tryptase, or provocation tests) remain useful to support the diagnosis of an IgE-mediated reaction.

Midazolam associated anaphylaxis is relatively rare and the risk factors associated with this event are actually unknown; however, it remains important to obtain a detailed allergic history and each surgical/endoscopic examination unit should be prepared to handle any situation or emergency that may occur.

(c) 2021 Codon Publications. Published by Codon Publications.

\section{Introduction}

Midazolam is a short-acting benzodiazepine with central nervous system depressing action, commonly used for conscious sedation for various procedures and for its pharmacologic properties. ${ }^{1}$ In literature, severe adverse reactions to this drug are described, ${ }^{2-7}$ but only in few cases positive allergological tests were demonstrated. ${ }^{5,7}$

The authors collected herein five clinical cases of different allergic reactions to midazolam demonstrated by positive allergological tests.

\section{Clinical Cases}

In the $1^{\circ}$ case, a male patient of 54 years old, apparently in good health and without allergic diseases, underwent an elective video laparoscopic cholecystectomy. He had normal routine preoperative tests. Intravenously premedication with midazolam $2 \mathrm{mg}$, propofol $150 \mathrm{mg}$, fentanyl $50 \mu \mathrm{g}$ and atracurium $35 \mathrm{mg}$ was performed with tracheal intubation, monitoring heart activity with electrocardiography (ECG), blood pressure (BP), arterial oxygen saturation $\left(\mathrm{SaO}_{2}\right)$ and end-tidal $\mathrm{CO}_{2}\left(\mathrm{ETCO}_{2}\right)$. After a few minutes, bradycardia with wide QRS and ST-segment elevation appeared on the ECG. Despite supplying him of oxygen with assisted ventilation and atropine administration, he presented a progressive decrease of heart frequency (HF) and BP until asystole. The resuscitation staff performed a cardiopulmonary resuscitation on the patient until there was recovery of a spontaneous circulation with peripheral pulses and the normalization of his BP and HF. A few hours later, the patient was healthy with stable vital signs.

In the $2^{\circ}$ case, a female patient of 24 years old with clinical history of allergic rhinitis and non-specific lipid transfer protein allergy, underwent an elective surgery for breast fibroadenoma. Routine preoperative tests were normal. Premedication consisted of locally administering lidocaine $200 \mathrm{mg}$ + ropivacaine $20 \mathrm{mg}$ and by intravenously midazolam $3 \mathrm{mg}+$ sufentanil $25 \mu \mathrm{g}$. Also, an oral prophylactic therapy with amoxicillin/clavulanic-acid, cetirizine, betamethasone, and ranitidine a few days before the surgery was performed. After $2 \mathrm{~h}$ from the surgical procedure, she presented with nausea, epigastric pain, and an itching widespread erythematous rash. Intravenously chlorphenamine and methylprednisolone were given with complete clinical remission.

The $3^{\circ}$ and $4^{\circ}$ cases involved two female patients of 46 and 49 years old with clinical history of ulcerative colitis + Basedow disease in the first one and eosinophilic colitis + nickel allergy in the second one, that respectively presented urticarial rash and edema/erythema localized on the infusion site of a sedation with midazolam $2 \mathrm{mg}+$ meperidine $50 \mathrm{mg}$ during an elective colonoscopy. Clinical remission was obtained after intravenously administering methylprednisolone and chlorphenamine in the first one and betamethasone in the second one. Later, they avoided the administration of these drugs or any type of sedation for surgical procedures.

In the $5^{\circ}$ case, a male patient of 19 years old with atopic dermatitis and familiar history of drug hypersensitivity reactions, presented an itching erythematous widespread rash + laryngeal edema/dyspnoea after a few minutes from an orthopaedic surgery performed by locally administering mepivacaine $350 \mathrm{mg}$ + ropivacaine $20 \mathrm{mg}$ and intravenously fentanyl $50 \mu \mathrm{g}$, midazolam $2 \mathrm{mg}$ and cefazoline $1 \mathrm{~g}$. Clinical remission was obtained after a few hours from the administration of hydrocortisone $1 \mathrm{~g}+$ dexamethasone $8 \mathrm{mg}+$ chlorphenamine $10 \mathrm{mg}$ intravenously and epinephrine with aerosol. Even here routine preoperative tests were normal.

All the patients were admitted to the Allergy Unit for an allergological evaluation. In every case that was evaluated, the culprit drugs included latex and chlorhexidine in the first case and penicillins/cefalosporins, respectively, in the second and fifth case. Skin prick tests (SPTs) and intradermal tests (IDTs), with the involved drugs, were performed at the concentrations described by Brockow and co-workers. ${ }^{8}$ Both allergological tests were conducted on the volar forearm of the patients; and for IDT, a test solution $(0.03-0.05 \mathrm{~mL})$ was injected intradermally into the skin to produce a bleb of $2-3 \mathrm{~mm}$ in diameter.

Histamine $(10 \mathrm{mg} / \mathrm{mL})$ was used as a positive control and saline solution $(0.9 \%)$ as a negative control. According to the criteria defined by the European Network for Drug Allergy (ENDA), ${ }^{9}$ the SPT was considered positive if a wheal of $\geq 3 \mathrm{~mm}$ in diameter was observed after $20 \mathrm{~min}$; while for IDT, if the size of the initial wheal increased by at least $3 \mathrm{~mm}$ in diameter and was surrounded by erythema after 20 min then it was considered positive. Moreover, specific IgEs (UniCAP-Phadia, Thermofisher, Uppsala, Sweden) were performed for:

- latex, chlorhexidine, morphine and pholcodine (these last drugs have a quaternary ammonium homologous to atracurium) in the first case;

- penicillin $\mathrm{G}$, penicillin $\mathrm{V}$, amoxicillin, ampicillin and cefaclor in the second case;

- cefaclor and latex in the fifth case. 


\begin{tabular}{|c|c|c|c|}
\hline $\mathrm{N}^{\circ}$ Case & $\begin{array}{c}\text { SPT } 5 \mathrm{mg} / \mathrm{mL} \\
(\mathrm{mm})\end{array}$ & $\begin{array}{l}\text { Initial wheal } \\
\text { IDT } 0.5 \mathrm{mg} / \mathrm{mL}\end{array}$ & $\begin{array}{l}\text { Reading wheal IDT } \\
0.5 \mathrm{mg} / \mathrm{mL}(\mathrm{mm})\end{array}$ \\
\hline $1^{\circ}$ & 0 & $3 \theta$ & $6 \otimes W$ and $15 \otimes E$ \\
\hline $2^{\circ}$ & 0 & $3 \theta$ & $8 \otimes W$ and $28 \otimes E$ \\
\hline $3^{\circ}$ & 0 & $3 \theta$ & $6 \otimes W$ and $12 \otimes E$ \\
\hline $4^{\circ}$ & 0 & 30 & $8 \otimes W$ and $15 \otimes E$ \\
\hline $5^{\circ}$ & 0 & $3 \theta$ & $8 \otimes W$ and $10 \otimes \mathrm{E}$ \\
\hline
\end{tabular}

$Q=$ diameter, $\mathrm{W}=$ wheal; $\mathrm{E}=$ erythema

IDT: Intradermal test; SPT: Skin prick test.

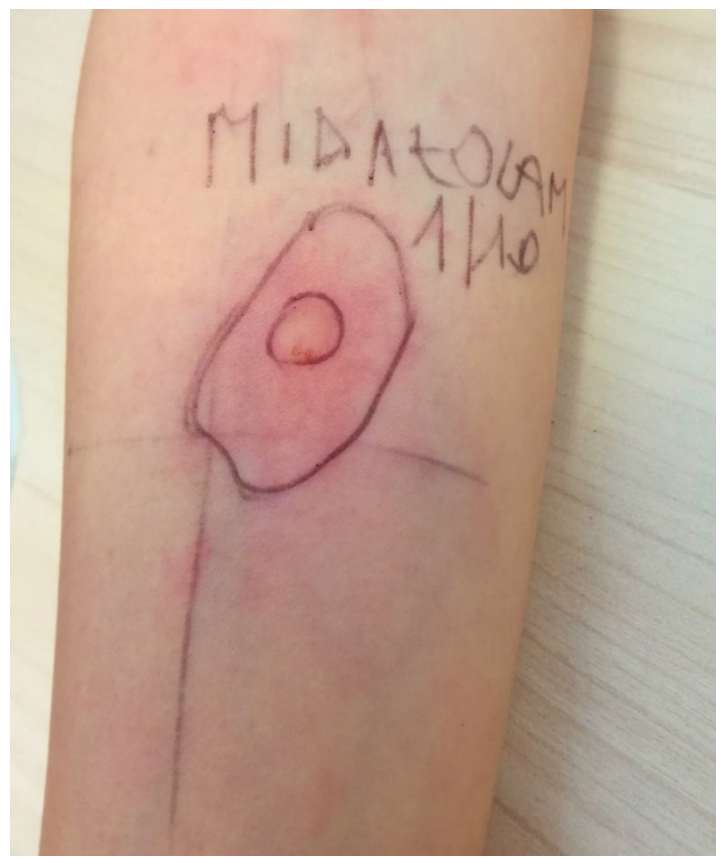

Figure 1 Intradermal test with midazolam at $0.5 \mathrm{mg} / \mathrm{mL}$ revealed a $8-\mathrm{mm}$ diameter wheal and a $28-\mathrm{mm}$ diameter erythema (Case 2).

In all the cases, only the IDT with midazolam at $0.5 \mathrm{mg} / \mathrm{mL}$ was positive. SPT at the maximum non-irritant concentration $(5 \mathrm{mg} / \mathrm{mL})$ resulted negative. Allergological tests performed in 10 healthy controls with negative results supported the diagnosis. In Table 1 all the positive reactions of the skin tests with midazolam are represented. In Figure 1 , the skin reaction during the IDT of the second case is represented.

\section{Discussion}

The $1^{\circ}$ case represents a case of cardiorespiratory arrest in a patient with demonstrated IgE-mediated allergy to midazolam. Ates et al. ${ }^{10}$ described a case of acute coronary syndrome during a transurethral prostatectomy (TUR-P) after intravenously administering midazolam injection with increment of serum troponin level and tryptase that was suggestive of a Kounis syndrome; but no allergological tests were performed. The patient showed clinical (bradycardia, cardiorespiratory arrest, hypotension) and electrocardiographic signs (sinus bradycardia, ST-segment elevation with wide $\mathrm{QRS}$ ), but none of the laboratory or imaging signs were able to confirm the syndrome. ${ }^{11}$ In conclusion, the first case could be a suspected Kounis syndrome in a patient with demonstrated IgE-mediated allergy to midazolam.

The $2^{\circ}$ and $5^{\circ}$ cases are two systemic reactions with involvement of the skin and gastrointestinal/respiratory system during elective surgeries in two patients with clinical history of atopia. In both the cases, the positive results of the allergological tests were suggestive of an IgEmediated allergy to this drug.

In the $3^{\circ}$ and $4^{\circ}$ cases, the authors described local skin reactions in correspondence with the infusion site of midazolam during the execution of a colonoscopy. In both cases, the patients had a history of gastrointestinal diseases (respectively, ulcerative colitis and eosinophilic colitis) but the laboratory tests were normal at the time of the endoscopic procedure.

In literature, only a few cases described a midazolam hypersensitivity confirmed by positive allergological tests. ${ }^{5-7}$ Therefore, midazolam is often considered a safe drug, because it does not have any active metabolites, in rare cases, it could cause different types of allergic adverse reactions: from severe anaphylaxis with cardiorespiratory arrest to simple local skin reactions.

Skin tests remain the first line in the diagnosis of an immediate-type hypersensitivity to midazolam; even if they could lose in sensitivity with increasing latency from the event. In particular, IDTs appear here to be more sensitive than SPTs to detect an IgE-mediated allergy to this drug. However, the concentration of $0.5 \mathrm{mg} / \mathrm{mL}$ used herein for IDTs recommended by current guidelines of ENDA and the European Academy of Allergy and Clinical Immunology (EAACl) drug allergy interest groups, might not rule out some false-positive reactions due to an irritant effect of the drug. In a study conducted by N. Hagau et al. ${ }^{12}$ using more clustered concentrations of the drug rather than those resulting from decimal dilutions, they identified that the maximum non-irritant concentration for IDT with midazolam should be considered $0.25 \mathrm{mg} / \mathrm{mL}$ instead of 0.5 $\mathrm{mg} / \mathrm{mL}$; since it has been found to be less associated with false positive reactions. ${ }^{12}$ However, the authors decided to follow the indications of ENDA/EAACl guidelines since the allergological tests (SPTs and IDTs) performed with the same concentration of the studied patient group showed negative results in their control group. Moreover, especially in the $1^{\circ}, 2^{\circ}$, and $5^{\circ}$ cases, clinical histories of patients seem highly suggestive of an IgE-mediated reaction to this drug; while in the $3^{\circ}$ and $4^{\circ}$ cases, an irritant effect of the drug during intravenous infusion cannot be excluded. For this reason, in the doubt cases, it remains useful to perform other allergological tests (e.g., basophil activation tests [BATs] or provocation tests) to support the diagnosis of a possible IgE-mediated reaction. In particular, the BAT should be used complementary to skin tests in patients with perioperative anaphylactic or anaphylactoid reactions, especially if it is supposed an IgE-mediated mechanism and skin tests are inconclusive. However, a recent study about the utility of this test to assess perioperative 
anaphylactic reactions showed a discrepancy between the number of positive reactions in skin testing performed with many perioperative substances (analgesics, antibiotics, local anesthetics, narcotics included midazolam and neuromuscular blocking agents) and the number of positive results in BAT. According to the authors, this could be explained by non-lgE mediated mechanisms or by irritative reactions of drugs itself or by a low sensitivity of the test. Conversely, BAT seems to have a good specificity and a positive reaction with a high activation of basophils and identifies the culprit agent in perioperative hypersensitivity reactions with high probability. ${ }^{13}$ Moreover, in these cases also a serum tryptase assay is always recommended to evaluate the causative symptoms of an anaphylactic reaction if it is suspected.

\section{Conclusion}

Midazolam-associated anaphylaxis is relatively rare and risk factors associated to this event are actually unknown; however, it remains important to obtain a detailed allergic history and each surgical/endoscopic examination unit should be prepared to handle any situation or emergency that may occur.

All the patients present in this case series have given the consent for publication of their identifiable details.

\section{Acknowledgments}

The authors are responsible for the content and the writing of this article.

\section{Conflicts of interest}

The authors declare that they have no conflicts of interest.

\section{Funding}

The authors declare that no funding was received for the present case series.

\section{References}

1. Möhler H, Fritschy JM, Rudolph U. A new benzodiazepine pharmacology. J Pharmacol Exp Ther. 2002;300(1):2-8. https://doi. org/10.1124/jpet.300.1.2
2. Shrivastava S. An experience with midazolam anaphylactoid reaction. J Anesth. 2012;26(4):642-623. https://doi. org/10.1007/s00540-012-1386-6

3. Shin JG, Hwang JH, Lee BS, Park HJ, Lee SH, Lee JN, et al. A case of midazolam anaphylaxis. Clin Eendosc. 2014;47(3):262265. https://doi.org/10.5946/ce.2014.47.3.262

4. Ayuse T, Kurata S, Ayuse T. Anaphylactoid-like reaction to midazolam during oral and maxillofacial surgery. Anesth Prog. 2015;62(2):64-65. https://doi.org/10.2344/0003-3006-62.1.64

5. Bernardini R, Bonadonna P, Catania P, Passalacqua G. Perioperative midazolam hypersensitivity in a seven-year-old boy. Pediatr Allergy Immunol. 2017;28(4):400-401. https://doi. org/10.1111/pai.12717

6. Hwang JY, Jeon YT, Na HS, Lee JH, Choi SJ, Jung SH. Midazolam hypersensitivity during the transportation to theater - A case report. Korean J Anesthesiol. 2010;59(Suppl):S1-S2. https:// doi.org/10.4097/kjae.2010.59.S.S1

7. Jeon YS, Shim J, Jun EH, Choi ST, Jung HS. Midazolam anaphylaxis during general anesthesia: A case report. Medicine (Baltimore). 2019;98(41):e17405. https://doi.org/10.1097/ MD.0000000000017405

8. Brockow K, Garvey LH, Aberer W, Atanaskovic-Markovic M, Barbaud A, Bilo MB, et al. ENDA/EAACI Drug Allergy Interest Group. Skin test concentrations for systemically administered drugs - An ENDA/EAACI Drug Allergy Interest Group position paper. Allergy. 2013;68(6):702-712. https://doi.org/10.1111/ all.12142

9. Brockow K, Romano A, Blanca M, Ring J, Pichler W, Demoly P, et al. General considerations for skin test procedures in the diagnosis of drug hypersensitivity. Allergy. 2002;57:45-51. https://doi.org/10.1046/j.0105-4538.2001.00001.x-i8

10. Ateș $\mathrm{AH}$, Kul S. Acute coronary syndrome due to midazolam use: Kounis syndrome during a transurethral prostatectomy. Turk Kardiyol Dern Ars. 2015;43(6):558-561. https://doi. org/10.5543/tkda.2015.44567

11. Kounis NG. Kounis syndrome: An update on epidemiology, pathogenesis, diagnosis and therapeutic management. Clin Chem Lab Med. 2016;54(10):1545-1559. https://doi. org/10.1515/cclm-2016-0010

12. Hagau N, Bologa RO, Indrei CL, Longrois D, Dirzu DS, GhermanIonica N. Maximum non-reactive concentration of midazolam and ketamine for skin testing study in non-allergic healthy volunteers. Anaesth Intensive Care. 2010;38(3):513-518. https:// doi.org/10.1177/0310057X1003800316

13. Eberlein $B$, Wigand $S$, Lewald $H$, Kochs E, Ring J, Biedermann $T$, et al. Utility of basophil activation testing to assess perioperative anaphylactic reactions in real-world practice. Immun Inflamm Dis. 2017;5(4):416-420. https://doi.org/10.1002/ iid3.175 